CASE REPORT

\title{
Malignant potential in a Brunner's gland hamartoma
}

\author{
M J Brookes, S Manjunatha, C A Allen, M Cox
}

Postgrad Med J 2003;79:416-417

Brunner's gland hamartomas are rare tumours of the duodenum. These lesions have previously been described as being benign, with no malignant potential. A case report is presented of a Brunner's gland hamartoma, whose histology revealed a focus of well marked epithelial dysplasia. This case suggests a dysplastic stage in the natural history of Brunner's gland hamartoma, and questions the malignant potential of these lesions.

$\mathrm{B}$ unner's gland hamartomas are rare duodenal tumours and an unusual cause of gastrointestinal bleeding. Since the first description of a Brunner's gland hamartoma in 1876 there has been controversy to the aetiology and pathogenesis of this lesion. Several case reports have described these lesions as non-dysplastic and entirely benign. However a case report is presented, representing a Brunner's gland hamartoma with a dysplastic focus. This case report illustrates that these lesions do appear to become dysplastic and raises the question of malignant potential.

\section{CASE REPORT}

A 79 year old white man presented with a collapse and melaena. He had a history of stable angina for three years, mild chronic obstructive pulmonary disease, and a previous transient ischaemic attack. He was taking $75 \mathrm{mg}$ of aspirin daily and took minimal alcohol. Examination revealed a soft non-tender abdomen and a blood pressure of 114/75 mm Hg, with no significant postural drop. Haematological investigations revealed anaemia, with a haemoglobin concentration of $92 \mathrm{~g} / \mathrm{l}$. On the second day the haemoglobin dropped to $72 \mathrm{~g} / \mathrm{l}$ and an upper gastrointestinal endoscopy was performed.

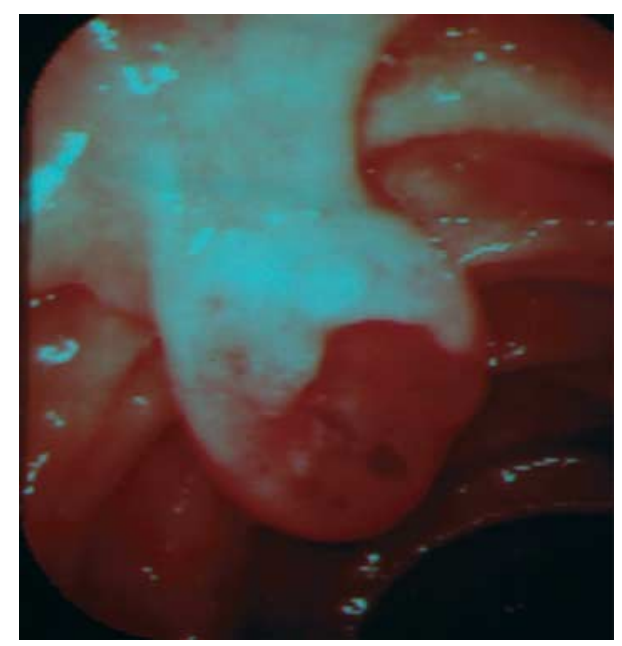

Figure 1 Endoscopic view of pedunculated Brunner's gland hamartoma in the second part of the duodenum.
At endoscopy a $2 \mathrm{~cm}$ pedunculated lesion, with an ulcerated crater, was noted in the second part of the duodenum (fig 1). There were no signs of active bleeding and the lesion was biopsied at this stage. The patient was transfused and subsequently maintained his haemoglobin.

Histology revealed a submucosal lesion with complex, crowded architecture and features consistent with a Brunner's gland hamartoma. However, in the deeper layers of the specimen, some nuclear atypia with occasional mitotic figures was seen. Given these findings a repeat endoscopy was performed and the lesion was removed by snare polypectomy using coagulation current.

Histology of the resected specimen revealed it to predominantly composed of lobules of relatively bland appearing Brunner's glands. However throughout the specimen there were foci of obvious dysplasia with some foci showing more pronounced cytological changes (fig 2).

\section{DISCUSSION}

Duodenal tumours, both malignant and benign, are rare. Brunner's gland hamartomas and adenomas account for up to $1 \%$ of small bowel tumours. ${ }^{1}$ Normal Brunner's glands are alkaline mucus-secreting and are found predominantly in the duodenum, extending to the proximal jejunum. ${ }^{2}$ Brunner's gland hamartomas are most often found in the proximal duodenum $(57 \%),{ }^{2}$ representing the normal Brunner's gland distribution. The majority are pedunculated $(88 \%),{ }^{2}$ and are commonly $1-2 \mathrm{~cm}$ in diameter. Clinical presentation is variable, and indeed many are asymptomatic and incidental findings. A recent series revealed the commonest presentations, in symptomatic patients, were gastrointestinal haemorrhage $(37 \%)$ and obstructive symptoms $(37 \%){ }^{3}$ Most symptomatic patients present in the fifth or sixth decade and there is equal sex distribution. Rarer presentations have included recurrent pancreatitis, ${ }^{4}$ obstructive jaundice, ${ }^{5}$ and biliary fistula. ${ }^{6}$ The diagnosis is usually made by radiographic and endoscopic techniques, and in the series by Levine et al, $92 \%$ were identified by small bowel studies. ${ }^{3}$ At endoscopy the classical findings are of a pedunculated polyp in the duodenal

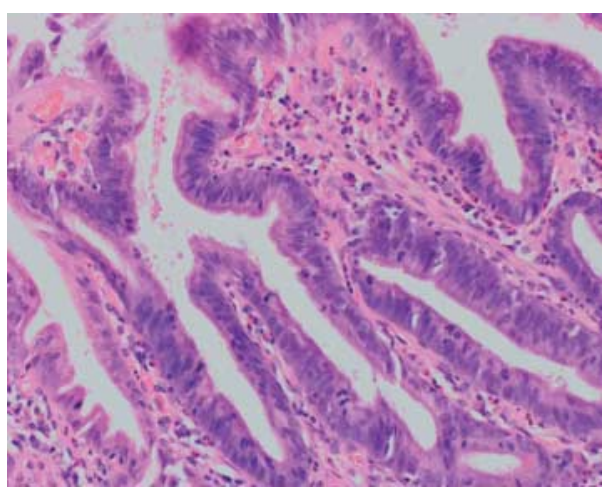

Figure 2 Photomicrograph of resected specimen showing well marked epithelial dysplasia (haematoxylin and eosin, original magnification $\times 200$ ). 
bulb, though $11 \%$ can be sessile. ${ }^{3}$ Histology classically reveals normal overlying mucosa and a well circumscribed lesion within the submucosa. This tends to contain normal Brunner's glands with glandular lobulation with intervening bands of paucicellular fibrous tissue. The Brunner's glands have small, oval, basally orientated nuclei and apical mucinuos cytoplasm, staining positive with periodic acid Schiff. Series and case reports, in the literature, comment on the considerable variation in nomenclature from Brunner's gland hyperplasia to adenoma to hamartoma. From reviewing the published literature it is clear that no previously examined Brunner's case reports or series comment on the entirely benign nature of these lesions and many suggest conservative management in asymptomatic patients.

In conclusion, this case illustrates the potential for dysplasia in these lesions, previously felt to be entirely benign, and raises the question of potential malignant transformation. Indeed there appears to be a continuum between Brunner's gland hyperplasia, hamartoma and adenoma, with a potential for dysplastic changes. Clearly, from the benign nature of Brunner's gland hamartoma in all the previous case reports, these dysplastic changes appear to be a rare phenomenon. However given the findings in this case it would be dangerous to presume that all Brunner's gland hamartomas are non-dysplastic and non-malignant.

\section{Authors' affiliations}

M J Brookes, Department of Gastroenterology, City General Hospital, Stoke-on-Trent

S Manjunatha, M Cox, Department of Gastroenterology, Manor Hospital, Walsall

C A Allen, Department of Histopathology, Manor Hospital, Walsall

Correspondence to: Dr Brookes; mbro453398@aol.com

Submitted 27 January 2003

Accepted 28 March 2003

\section{REFERENCES}

1 Botsford TW, Crowe P, Crocker D. Tumours of the small intestine. Am J Surg 1962;103:358-65.

2 Walden DT, Marcon NE. Endoscopic injection and polypectomy for bleeding Brunner's gland hamartoma: case report and expanded literature review. Gastrointest Endosc 1998;48:403-7.

3 Levine JA, Burgart $\mathrm{W}$, Batts KP, et al. Brunner's gland hamartomas: clinical presentation and pathological features of 27 cases. Am J Gastroenterol 1995;90:290-4.

4 Scholz HG. Recurrent acute pancreatitis: a complication of Brunneromas. Leber Magen Darm 1976;6:300-2.

5 Skellenger ME, Kinner BM, Jordan PH Jr. Brunner's gland hamartomas can mimic carcinoma of the head of the pancreas. Surg Gynaecol Obstet 1983:156:774-6.

6 Hedges AR. Hamartoma of Brunner's gland causing pyloric obstruction and a biliary fistula. Acta Chir Scand 1988;154:475-6.

\section{IMAGES IN MEDICINE.}

\section{A gangrenous foot}
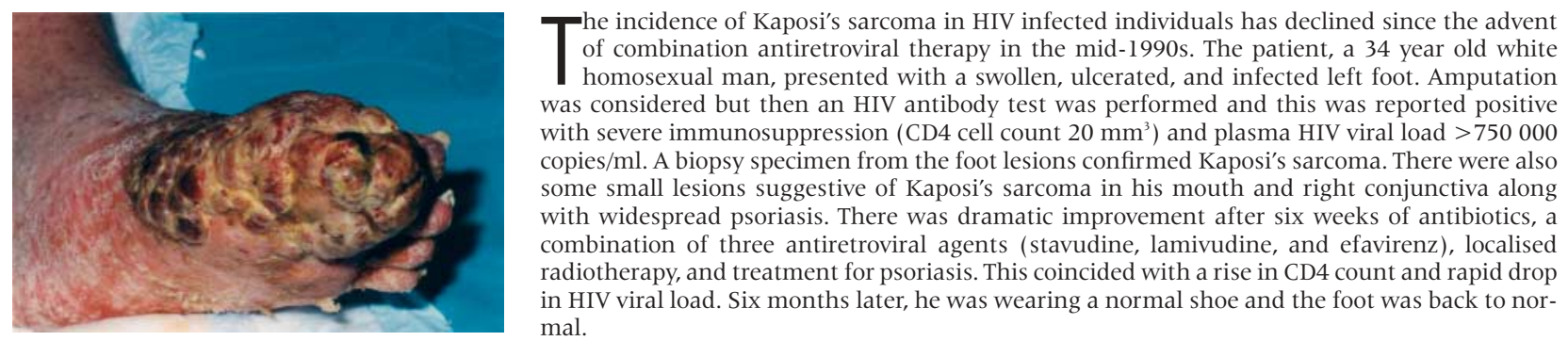

G Singh 